\title{
Low cost temperature- and strain-insensitive twist sensor based on hybrid fiber grating structure
}

\author{
XI Guo, ${ }^{1}$ Zhikun XING, ${ }^{1}$ Huabao QIn, ${ }^{1}$ QIZHen Sun, ${ }^{1}$ Hushan Wang, ${ }^{2}$ Deming \\ LIU, ${ }^{1}$ LIN ZHANG, ${ }^{3}$ AND ZHIJUN YAN, ${ }^{1,2,3},{ }^{*}$ \\ ${ }^{1}$ School of Optical and Electronic Information, National Engineering Laboratory for Next Generation Internet Access System, Huazhong \\ University of Science and Technology, Wuhan 430074, China \\ ${ }^{2}$ State Key Laboratory of Transient Optics and Photonics, Xi'an Institute of Optics and Precision Mechanics, Chinese Academy of Sciences, Xi'an \\ 710119, China \\ ${ }^{3}$ Aston Institute of Photonic Technologies, Aston University, Birmingham, UK, B4 7ET \\ *Corresponding author: yanzhijun@gmail.com
}

Received XX Month XXXX; revised XX Month, XXXX; accepted XX Month XXXX; posted XX Month XXXX (Doc. ID XXXXX); published XX Month XXXX

\begin{abstract}
We proposed and experimentally demonstrated a temperature- and strain-insensitive twist sensor based on a hybrid fiber grating structure, in which the hybrid grating structure is constructed with a $45^{\circ}$-tilted fiber grating (45 ${ }^{\circ}$-TFG) and a chirped fiber Bragg grating (CFBG) UV-inscribed in a single mode fiber (SMF) in series. The sensing performance has been evaluated by experimental and numerical analyses which are in good consistency. The experimental results show the hybrid grating structure based twist sensor has a maximum twist sensitivity up to $15.037 \mathrm{~dB} / \mathrm{rad}$. Moreover, due to the invariability of the fiber birefringence and the state of polarization of the input light, such sensor has intrinsically low temperature and strain sensitivities of $7.86 \times 10^{-3} \mathrm{~dB} /{ }^{\circ} \mathrm{C}$ and $6.7 \times 10^{-5} \mathrm{~dB} / \mu \varepsilon$, corresponding to the maximum twist measurement error resulting from temperature and strain of $5.2 \times 10^{-4} \mathrm{rad} /{ }^{\circ} \mathrm{C}$ and $4.5 \times 10^{-6} \mathrm{rad} / \mu \varepsilon$, respectively.
\end{abstract}

http://dx.doi.org/10.1364/AO.99.099999

\section{INTRODUCTION}

Twist sensors have attracted growing research interest for security monitoring of civil engineering, industrial application, and arms of humanoid robots because twist is a significant mechanical parameter that reflects the deformation and stress condition of the monitored structures. There are two types of twist sensors: electronics and optics. The conventional twist sensors are mainly based on electromagnetic phenomena [1] and electrical methods [2], which usually have bulky heavy structures and cross-sensitivity problems. Compared with the electrical twist sensors, the optical fiber twist sensors are regarded as another promising candidates because of their inherent merits, such as electromagnetic interference-free, compact size, light weight, and distributed measurement over a long distance, which are superior in practical applications. Up to now, various types of optical fiber twist sensors have been extensively investigated and developed. According to the different configurations, optical fiber twist sensors can be mainly categorized into the following types: fiber gratings [310] and fiber interferometers [11-18]. For example, a considerable amount of fiber gratings including fiber Bragg gratings (FBGs) [3-5], chirped fiber Bragg gratings (CFBGs)
[6], phase shifted FBG [7] and long period gratings (LPGs) [810] have been employed to construct optical fiber twist sensors with compact structures. Meanwhile, the fiber interferometer based twist sensors also have great potential due to their high sensitivity and fast response, which can be realized by Sagnac interferometer (SI) [11-13], multi-mode interferometers (MMI) [14,15], Mach Zehnder Interference (MZI) [16,17] and Solc filters [18]. However, most of fiber grating based twist sensors suffer from temperature and strain cross-sensitivity problems and relatively low twist sensitivity. And fiber interferometer based sensors always need comparatively complex signal demodulation system and special optical fibers or structures, which might limit their potential applications.

A polarization detection process is a simple and effective method to realize twist measurement. When a beam of linear polarized light transmits in an optical component under twist, the output intensity after the polarization analyzer would change with the twist. Therefore, the twist effect can be detected by the intensity variation. In this paper, we propose and demonstrate a hybrid fiber grating structure based temperature- and strain-insensitive twist sensor, in which the hybrid grating structure is fabricated by inscribing a section of $45^{\circ}$-tilted fiber grating $\left(45^{\circ}-\mathrm{TFG}\right)$ and chirped fiber Bragg 
grating $(\mathrm{CFBG})$ in the single mode fiber (SMF) in series. The $45^{\circ}$-TFG has been implemented as an ideal in-fiber polarizing component since proposed in 2005 [19], due to its prominent advantages, including high polarization-extinction-ratio (PER), low insertion loss, and nice compatibility with other fiber components. The CFBG which acts as a fiber reflector promotes the sensor a compact single lead-in structure.
Compared with the reported twist sensors working in
reflection [20-22], the proposed method has much simpler configuration and higher sensitivity. The experimental results show that the proposed sensor exhibits a high twist sensitivity up to $15.037 \mathrm{~dB} / \mathrm{rad}$, which show good consistency with the numerical analysis results. Furthermore, the proposed twist sensor has ultra-low temperature and strain sensitivities of $7.86 \times 10^{-3} \mathrm{~dB} /{ }^{\circ} \mathrm{C}$ and $6.7 \times 10^{-5} \mathrm{~dB} / \mu \mathrm{\varepsilon}$, corresponding to the maximum twist measurement error resulting from temperature and strain of $5.2 \times 10^{-4} \mathrm{rad} /{ }^{\circ} \mathrm{C}$ and $4.5 \times 10^{-6} \mathrm{rad} / \mu \varepsilon$, respectively.

\section{DEVICE PRINCIPLE AND THEORETICAL ANALYSIS}

The schematic diagram of the hybrid fiber grating structure based twist sensor is shown in Fig. 1, which is constituted by inscribing a section of $45^{\circ}$-TFG (PER of $33 \mathrm{~dB}$ ) and CFBG (reflectivity of $25 \mathrm{~dB}$ ) in the SMF with a segment of fiber cavity. In the sensor configuration, the $45^{\circ}$-TFG is used as an ideal fiber polarizer to convert the light launched from the amplified spontaneous emission (ASE) light source into linearly polarized light, and a fiber polarization analyzer for the light reflected by the CFBG simultaneously. When the fiber cavity between the $45^{\circ}$-TFG and CFBG is subjected to twist, the polarization state of the light transmitting in the fiber will be changed, resulting in the output intensity variation. In this case, the twist angle applied on the fiber cavity can be deduced by simply monitoring the intensity change. Thus, based on the proposed hybrid grating structure, we demonstrate an optical fiber twist sensor.

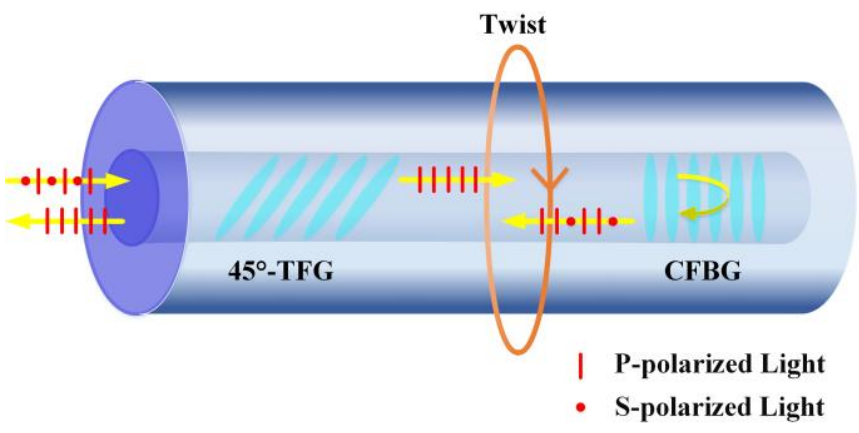

Fig. 1. Schematic diagram of the hybrid fiber grating structure based twist sensor.

For a fiber under linear twist $\theta$ shown in Fig.1, without any change of other optical components, the Jones matrix of the twisted fiber can be given as [23]:

$$
\mathrm{F}_{L}=\left[\begin{array}{cc}
\cos \eta-i \frac{\delta_{L}}{2} \frac{\sin \eta}{\eta} & \frac{\delta_{C}}{2} \frac{\sin \eta}{\eta} \\
-\frac{\delta_{C}}{2} \frac{\sin \eta}{\eta} & \cos \eta+i \frac{\delta_{L}}{2} \frac{\sin \eta}{\eta}
\end{array}\right]
$$

Here:

$$
\begin{array}{r}
\delta_{L}=\frac{2 \pi}{\lambda} \cdot \Delta n \cdot L \\
\delta_{C}=2\left(1-\frac{g}{2}\right) \cdot \theta \\
\eta=\sqrt{\left(\frac{\delta_{L}}{2}\right)^{2}+\left(\frac{\delta_{C}}{2}\right)^{2}}
\end{array}
$$

$\delta_{\mathrm{L}}$ and $\delta_{\mathrm{C}}$ are the phase terms pertaining to the linear and circular birefringence of the corresponding fiber, respectively. $\Delta \mathrm{n}$ is the linear birefringence, $\lambda$ is the operating wavelength, and $g=0.16$ is a constant parameter for the conventional silica fiber. In this work, due to the low linear birefringence of the SMF which is about $10^{-7}$, the twist applied onto the SMF could be considered as a strong twist, in which case, the circular birefringence is dominant [24]. Therefore, Eq. (1) can be simplified as:

$$
\mathrm{F}_{L}(\theta)=\left[\begin{array}{cc}
\cos \frac{\delta_{C}}{2} & \sin \frac{\delta_{C}}{2} \\
-\sin \frac{\delta_{C}}{2} & \cos \frac{\delta_{C}}{2}
\end{array}\right]=\left[\begin{array}{cc}
\cos (0.92 \theta) & \sin (0.92 \theta) \\
-\sin (0.92 \theta) & \cos (0.92 \theta)
\end{array}\right]
$$

In addition, the Jones matrix of the $45^{\circ}-\mathrm{TFG}$ and $\mathrm{CFBG}$ can be expressed as:

$$
\begin{gathered}
\mathrm{P}_{45-T F G}=\left[\begin{array}{cc}
1 & 0 \\
0 & 0
\end{array}\right] \\
M_{C F B G}=\left[\begin{array}{cc}
1 & 0 \\
0 & -1
\end{array}\right]
\end{gathered}
$$

By using the transfer matrix method, the proposed hybrid fiber grating structure based twist sensor can be described as:

$$
M=\mathrm{P}_{45-T F G} \cdot \mathrm{F}_{L}(-\theta) \cdot M_{C F B G} \cdot \mathrm{F}_{L}(\theta) \cdot \mathrm{P}_{45-T F G}
$$

The output intensity is shown as:

$$
I_{\text {out }}=\cos ^{2}(1.84 \theta)
$$

Assuming a beam of non-polarized light enters into the proposed structure, according to Eq. (7), the output intensity is a sinusoidal function with the twist angle, and the twist sensitivity is independent of the twisted fiber length, environmental temperature and strain.

\section{RESULTS AND DISCUSSION}

The twisting experimental setup is shown in Fig. 2, in which a segment of fiber cavity between the $45^{\circ}$-TFG and CFBG is clamped by a fiber holder on one side and a fiber rotator with an engraved dial on the other end. To eliminate the measurement error induced by bending, a pre-strain is applied on the fiber to keep the twist section straight. An ASE source is used as non-polarized light source and is launched into the port 1 of the circulator and the output from port 3 is recorded by the optical power meter. 


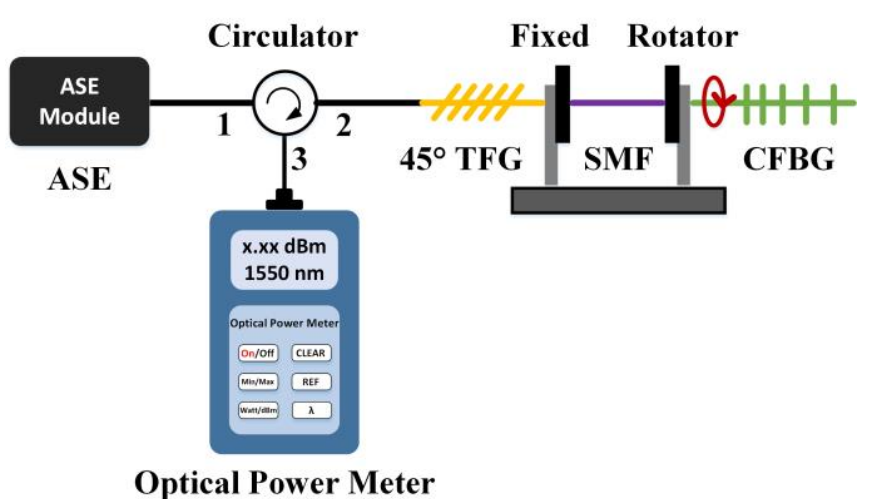

Fig. 2. Experimental setup for twist measurement.

During the twist measurement, one end of the fiber was rotated from $0^{\circ}$ to $360^{\circ}$ with an increment of $5^{\circ}$ and the light intensity was measured by the optical power meter. We conducted the experiment for three times to show the repeatability. The experimental result is plotted in Fig. 3(a), which presents the relationship between the intensity and the applied twist angles. It is obvious that the intensity changes periodically with the twist angles as a sine-like function, and the sensor demonstrates good repeatability due to the slight standard deviation. As we can note, the change of each dip in amplitude may arise from the transmission loss of increasing
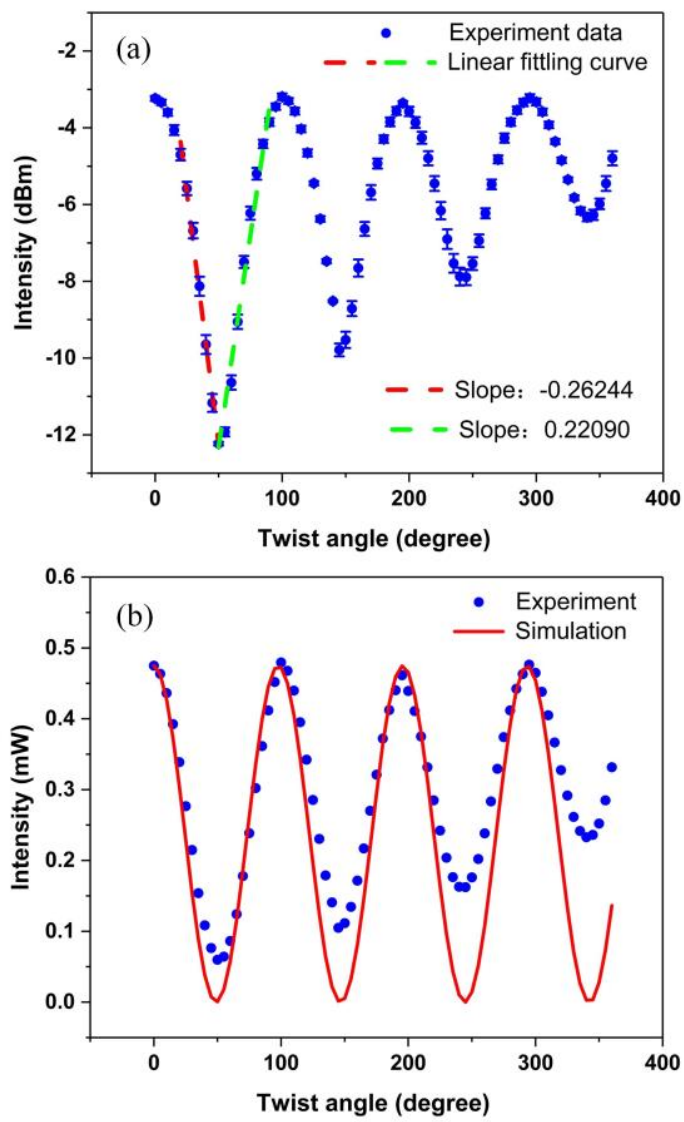
Fig. 3. Intensity variation with applied twist angle. (a) Twist
response with maximum sensitivity of $-15.037 \mathrm{~dB} / \mathrm{rad}$. (b) Comparison between simulated and experimental results. twist and residual stress or core ellipticity resulting from the practical fiber fabrication process. Therefore, the proposed sensor has the maximum twist sensitivity of $-15.037 \mathrm{~dB} / \mathrm{rad}$ in the region from $10^{\circ}$ to $50^{\circ}$, according to the slope of the fitting curve of -0.26244 . Figure 3 (b) depicts the calculated and measured results, which shows that the light intensity varies with the twist angles in a sinusoidal waveform and both the results have the same angle period. A comparison between our work and the previous fiber twist sensors by using intensity modulation is listed in Table 1 , in which the sensitivity of the proposed twist sensor is one order higher than that of the previous work.

Table 1. A Comparison of the Sensitivity Between Our Work and the Previous Reports

\begin{tabular}{cc}
\hline Reports & Twist Sensitivity \\
\hline This work & $15.037 \mathrm{~dB} / \mathrm{rad}$ \\
Yin et al. [6] & $0.1466 \mathrm{~dB} /(\mathrm{rad} / \mathrm{m})$ \\
Wang et al. [8] & $1.911 \mathrm{~dB} / \mathrm{rad}$ \\
Frazão et al. [13] & $0.687 \mathrm{~dB} / \mathrm{rad}$ \\
Sun et al. [18] & $1.2714 \mathrm{~dB} /(\mathrm{rad} / \mathrm{m})$ \\
\hline
\end{tabular}

From Eq. (7), the light intensity of the proposed sensor only depends on the twist angle, which is not affected by the sensor length under twisting, temperature and strain within a certain range. To verify this, we investigated experimentally the performance of the sensors with three different fiber lengths $(5 \mathrm{~cm}, 10 \mathrm{~cm}$, and $15 \mathrm{~cm})$ under twisting range from $0^{\circ}$ to $200^{\circ}$. As is revealed in Fig. 4, the twist sensors with three different fiber lengths show almost the same sensing results, since the standard deviations are small enough to be considered ignorable.

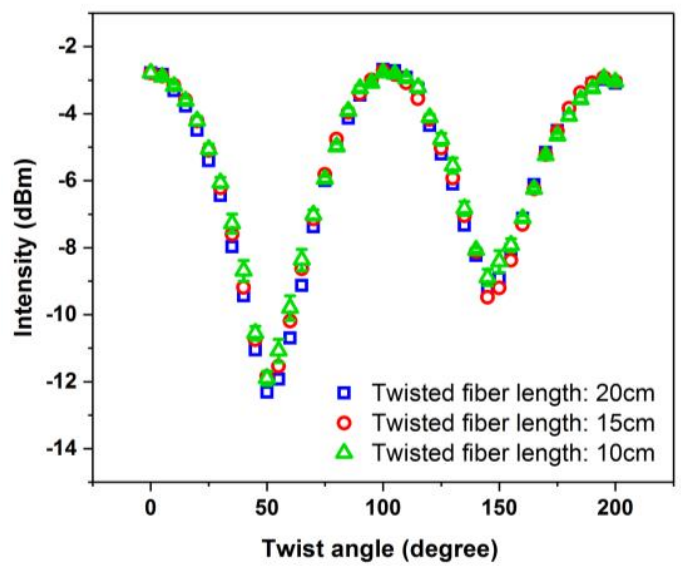

Fig. 4. Sensor performance with different twisted fiber length.

Furthermore, we have also investigated the cross sensitivities of temperature and strain. In experiment, the fiber cavity between the $45^{\circ}$-TFG and CFBG was subjected to a temperature controller with three different pre-twist angles $\left(50^{\circ}, 70^{\circ}\right.$ and $\left.100^{\circ}\right)$. The heating temperature was stepwise increased from $10{ }^{\circ} \mathrm{C}$ to $80{ }^{\circ} \mathrm{C}$ with an increment of $10{ }^{\circ} \mathrm{C}$. Figure 5(a) shows the measured intensity variation with different temperature levels, in which the intensity barely 
changes, and the maximum linear fitting coefficient is only $7.86 \times 10^{-3} \mathrm{~dB} /{ }^{\circ} \mathrm{C}$, corresponding to the maximum twist measurement error of $5.23 \times 10^{-4} \mathrm{rad} /{ }^{\circ} \mathrm{C}$. For the strain measurement, the increasing axial strain $(0 \sim 1000 \mu \varepsilon)$ was applied on the sensor by adjusting the translation stage. The strain was calculated from the elongation of the stretched fiber divided by its original length. The relationship between the output intensity and axial strain under different pre-applied twist angles $\left(50^{\circ}, 70^{\circ}\right.$ and $\left.100^{\circ}\right)$ is revealed in Fig. 5(b), in which the intensity almost remains stable. The strain sensitivity is only $6.7 \times 10^{-5} \mathrm{~dB} / \mu \varepsilon$, which contributes to the maximum twist measurement error of $4.03 \times 10^{-6} \mathrm{rad} / \mu \varepsilon$. This can be explained by the fact that when the fiber is subjected to the temperature and strain uniformly, the birefringence of the SMF hardly changes, leading to that the polarization state of the light transmitting in the sensor almost remains the same. As a result, the intensity keeps unchanged. As for the temperature and strain effect on $45^{\circ}-\mathrm{TFG}$ and $\mathrm{CFBG}$, in our
previous work [25], we have investigated the temperature
characteristics of $45^{\circ}$-TFG, which shows that the $45^{\circ}-\mathrm{TFG}$ is
thermally stable with sensitivity of $7.14 \times 10^{-3} \mathrm{~dB} /{ }^{\circ} \mathrm{C}$. And the
CFBG that we used in the experiment has nearly ignorable
intensity change resulting from temperature and strain since
CFBG has relatively wide bandwidth and small wavelength
shift. It is the same case with the $45^{\circ}$-TFG. Therefore, the proposed sensor can be considered insensitive to temperature and strain, which is an important characteristic in view of its application as a twist sensor.
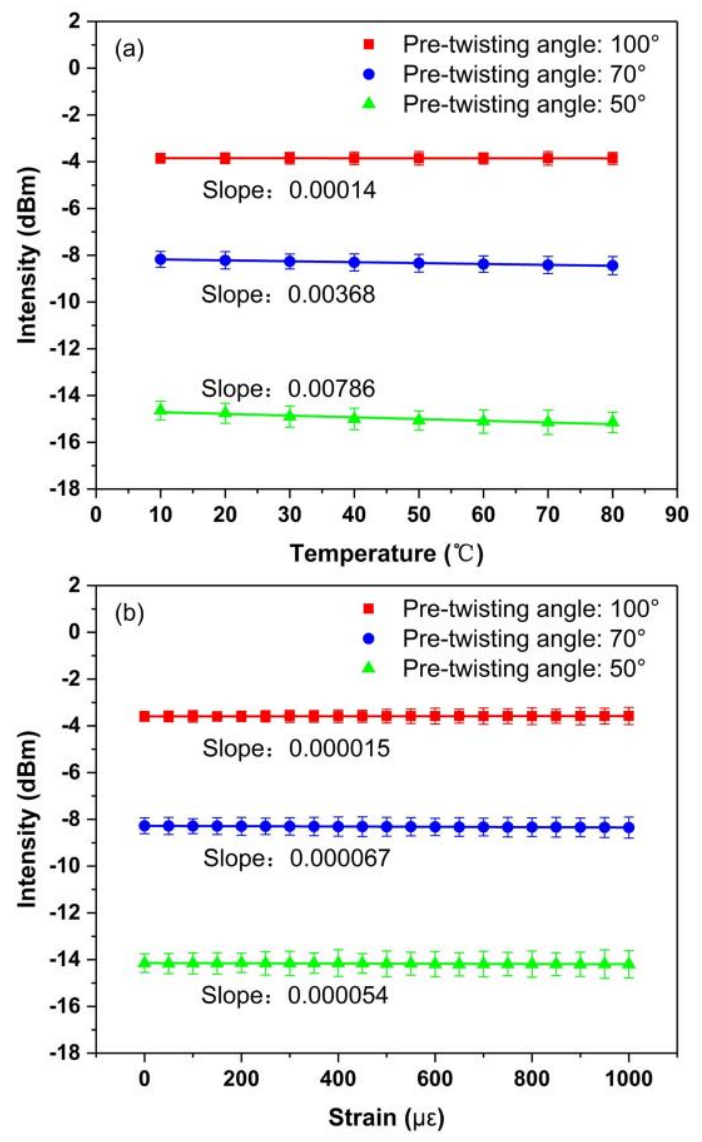

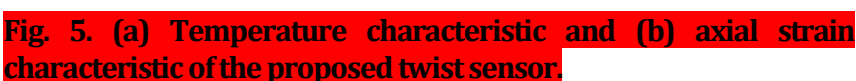

To investigate the sensor performance with different PER, three $45^{\circ}$-TFGs with PER of $6 \mathrm{~dB}, 15 \mathrm{~dB}$ and $33 \mathrm{~dB}$ were selected along with the CFBG (reflectivity of $25 \mathrm{~dB}$ ) to conduct the twist measurement. As revealed in Fig. 6, the results with PER of $15 \mathrm{~dB}$ and $33 \mathrm{~dB}$ are in good accordance, while the one with PER of $6 \mathrm{~dB}$ is less accurate. It can be inferred that the sensor based on the $45^{\circ}$-TFG with PER of $15 \mathrm{~dB}$ which is high enough to guarantee the function and accuracy can have good twist performance.

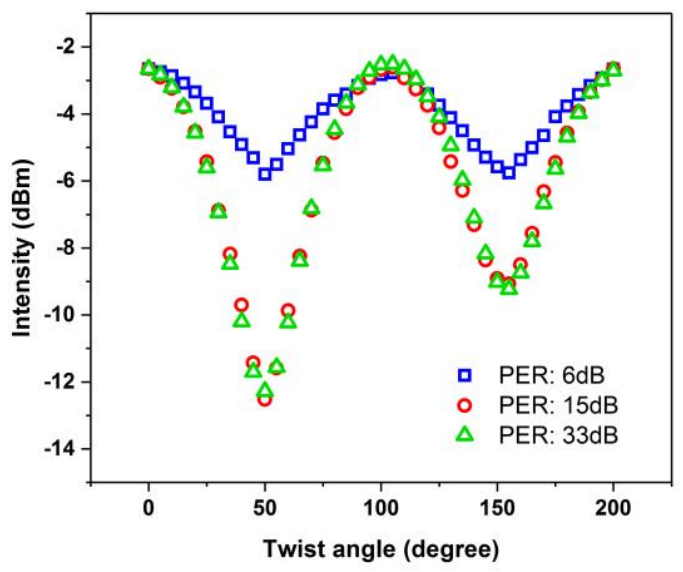

Fig. 6. Twist response with different PER of $45^{\circ}$-TFGs.

We also investigated the stability of the proposed sensor by measuring the twist response from $0^{\circ}$ to $360^{\circ}$ and $360^{\circ}$ to $0^{\circ}$, respectively. The result is plotted in Fig. 7. As shown in the figure, both intensities for the increasing and decreasing twist angles match very well, which demonstrates that the proposed sensor has good stability of its response with no evidence of hysteresis. This indicates the proposed twist sensor is desirable for real-time monitoring.

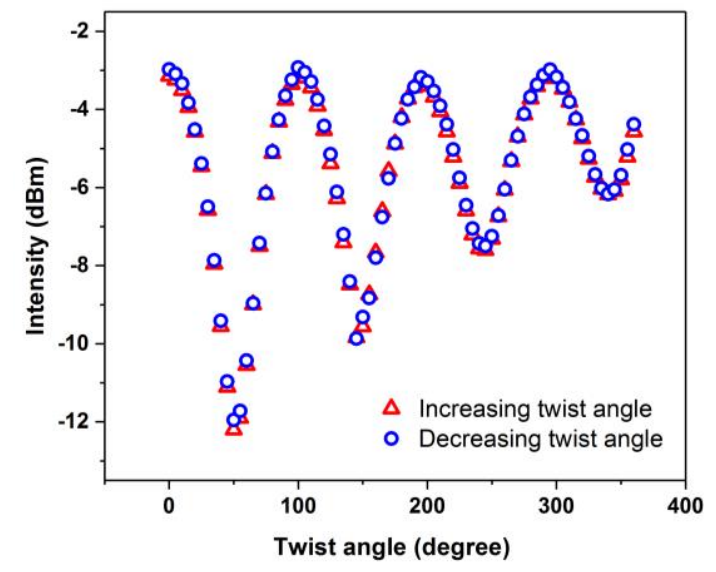

Fig. 7. Stability of the proposed twist sensor.

\section{CONCLUSION}


In summary, we propose and experimentally demonstrate a temperature- and strain-insensitive optical fiber twist sensor based on a hybrid $45^{\circ}-\mathrm{TFG}$ and $\mathrm{CFBG}$ structure. The experimental results show that the intensity changes as sinelike function with the twist angle, which agrees well with the theoretical analysis. In addition, based on the intensity modulation, the proposed twist sensor exhibits a high twist sensitivity up to $15.037 \mathrm{~dB} / \mathrm{rad}$, which is independent of the sensing fiber length within a certain range. Meanwhile, its temperature and strain cross-sensitivities are low to $7.86 \times 10^{-3}$ $\mathrm{dB} /{ }^{\circ} \mathrm{C}$ and $6.7 \times 10^{-5} \mathrm{~dB} / \mu \varepsilon$, respectively. The proposed twist sensor with prominent advantages such as extremely simple and robust structure, high sensitivity to twist and immunity to temperature and strain is highly desirable for modern smart structure monitoring applications.

Funding. National Natural Science Foundation of China (No. 61505244, 61605239); Fundamental Research Funds for the Central Universities, HUST: 2017KFYXJJ033; Science Fund for Creative Research Groups of the Nature Science Foundation of Hubei (NO. 2018CFA004); Major Projects of Technical Innovation of Hubei (NO. 2018AAA040).

\section{References}

1. D. Vischer and O. Khatib, "Design and development of high-performance torque controlled joints," IEEE Trans. Robot. Autom. 11, 537-544 (1995).

2. P. L. Fulmek, F. Wandling, W. Zdiarsky, G. Brasseur, and S. P. Cermak, "Capacitive sensor for relative angle measurement. Instrumentation and Measurement," IEEE Trans. Instrum. Meas. 51, 1145-1149 (2002).

3. F. Yang, Z. Fang, Z. Pan, Q. Ye, H. Cai, and R. Qu, "Orthogonal polarization mode coupling for pure twisted polarization maintaining fiber Bragg gratings," Opt. Express. 20, 28839-28845 (2012).

4. J. Wo, M. Jiang, M. Malnou, Q. Sun, J. Zhang, P. P. Shum, and D. Liu, "Twist sensor based on axial strain insensitive distributed Bragg reflector fiber laser," Opt. Express. 20, 2844-2850 (2012).

5. W. Yiping, M. Wang, and X. Huang, "In fiber Bragg grating twist sensor based on analysis of polarization dependent loss," Opt. Express. 20, 11913-11920 (2013).

6. B. Yin, H. Li, S. Feng, Y. Bai, Z. Liu, W. Peng, S. Liu, and S. Jian, "Temperature-independent and strain-independent twist sensor based on structured PM-CFBG," IEEE Photon. Technol. Lett. 26, 1565-1568 (2014).

7. B. Huang and X. Shu, "Ultra-compact strain- and temperature-insensitive torsion sensor based on a line-by-line inscribed phase-shifted FBG," Opt. Exp. 24, 17670-17679 (2016).

8. Y. Wang, X. Huang, and M. Wang, "Temperature insensitive birefringent LPG twist sensing based on the polarization properties," IEEE Photon. Technol. Lett. 27, 2367-2370 (2015).

9. Deng, M, Jinshan X, Zhe Z, Zhiyong B, Shen L, and Ying W, "Long period fiber grating based on periodically screw-type distortions for torsion sensing," Opt. Express. 25, 14308-14316 (2017).

10. Kang Y, Yange L, Zhi W, Yan L, Ya H, Hongwei Z, "Twist Sensor Based on Long Period Grating and Tilted Bragg Grating Written in Few-mode Fibers," IEEE Photonics Journal. 10, 99-108 (2018).

11.P. Zu, C. Chan, Y. Jin, T. Gong, Y. Zhang, L. Chen, and X. Dong, "A temperature-insensitive twist sensor by using low-birefringence photonic-crystal-fiber-based Sagnac interferometer," IEEE Photon. Technol. Lett. 23, 920-922 (2011).

12. B. Song, H. Zhang, Y. Miao, W. Lin, J. Wu, H. Liu, D. Yan, and B. Liu, "Highly sensitive twist sensor employing Sagnac interferometer based on PMelliptical core fibers," Opt. Express. 23, 15372-15379 (2015).

13

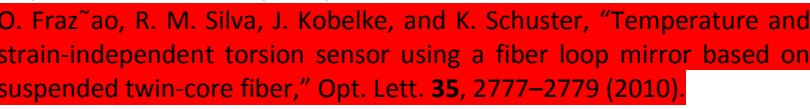

14.B. Song, Y. Miao, W. Lin, H. Zhang, J. Wu, and B. Liu, "Multi-mode interferometer-based twist sensor with low temperature sensitivity employing square coreless fibers," Opt. Exp. 21, 26806- 26811 (2013).

15.Xuexue $K$, Weigang $Z$, Yanxin Z, Jiang $Y$, Lei $C$, Lingxin $K$, "Intensitydemodulated torsion sensor based on thin-core polarization-maintaining fiber," Appl. Opt. 57, 3474-3478 (2018).

16. L. Chen, W. G. Zhang, L. Wang, H. Zhang, J. Sieg, Q. Zhou, L. Y. Zhang, B. Wang, and T. Y. Yan, "In-fiber torsion sensor based on dual polarized Mach-Zehnder interference," Opt. Express. 22, 31654-31664 (2014).

17. Qinjiang F, Jingdong Z, Chuangcan L, Iroegbu Paul, Guolu Y, and Lei L, "Intensity-modulated directional torsion sensor based on in-line optical fiber Mach-Zehnder interferometer," Opt. Lett. 43, 2414-2417 (2018).

18.Chunran, Sun, W. Muguang, and J. Shuisheng, "Experimental and theoretical study of the in- fiber twist sensor based on quasi-fan Solc structure filter," Opt. Express. 25, 19955-19965 (2017).

19. Kaiming Zhou, Xianfeng Chen, Lin Zhang, and lan Bennion, "High extinction ratio in-fiber polarizers based on $45^{\circ}$ tilted fiber Bragg gratings," Opt. Lett. 30, 1285-1287 (2005).

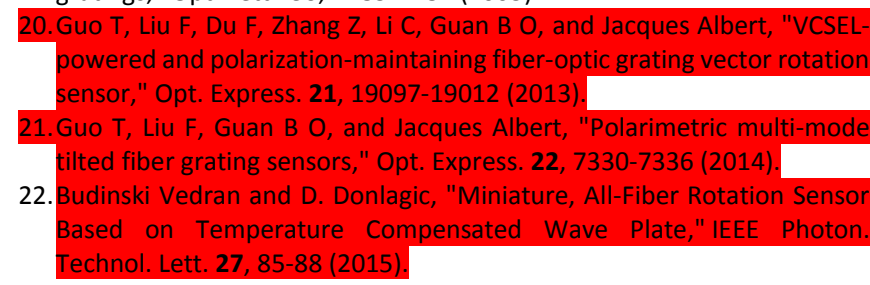

23. B. Ibarra-Escamilla, E. Kuzin, F. Gutierrez-Zainos, R. Tellez-Garcia, J. Haus, R. Rojas-Laguna, and J. EstudilloAyala, "Measurement of beat length in short low-birefringence fibers using the fiber optical loop mirror," Opt. Commun. 217, 211-219 (2003).

24.R. Ulrich and A. Simon, "Polarization optics of twisted single-mode fibers," Appl. Opt. 18, 2241-2251 (1979).

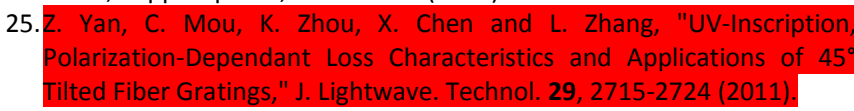

